International Journal of Modern Physics A

(C) World Scientific Publishing Company

\title{
GRAVITY AS BF THEORY PLUS POTENTIAL
}

\author{
KIRILL KRASNOV \\ School of Mathematical Sciences, University of Nottingham, Nottingham, NG7 2RD, UK \\ kirill.krasnov@nottingham.ac.uk \\ Received Day Month Year \\ Revised Day Month Year
}

\begin{abstract}
Spin foam models of quantum gravity are based on Plebański's formulation of general relativity as a constrained $\mathrm{BF}$ theory. We give an alternative formulation of gravity as $\mathrm{BF}$ theory plus a certain potential term for the B-field. When the potential is taken to be infinitely steep one recovers general relativity. For a generic potential the theory still describes gravity in that it propagates just two graviton polarizations. The arising class of theories is of the type amenable to spin foam quantization methods, and, we argue, may allow one to come to terms with renormalization in the spin foam context.
\end{abstract}

Keywords: Quantum gravity; spin foam models; modified gravity.

PACS numbers: 04.60.Pp, 04.50.Kd

\section{Introduction}

The starting point of one of the approaches to quantum gravity - spin foam models - is Plebański's 1 formulation of general relativity as BF theory supplemented with the so-called simplicity constraints for the B-field. There are several similar versions of this formulation, namely those based on the (complexified) rotation $\mathrm{SO}(3)$ and Lorentz $\mathrm{SO}(1,3)$ groups. Currently the most popular is the Lorentz group-based version, see e.g. 2, whose advantage is that no additional reality conditions on the B-field need to be imposed. The new spin foam models of (Riemannian signature) quantum gravity, see e.g. 3 , can be seen to be directly motivated by this version of Plebański theory.

On the other hand, the original 11 rotation group-based formulation works with self-dual quantities and therefore, in case one wants to describe metrics of Lorentzian signature, necessarily involves complex quantities - the price to pay for its notable algebraic simplicity. However, so far no simple spin foam model motivated by the self-dual Plebański theory was proposed - even in the case of Riemannian signature spacetimes where no problems with reality conditions would exist. See, however, 4 . This has to do with our present rather poor understanding of how to translate the self-dual simplicity constraints into the discrete setting of spin foams.

The main aim of this contribution is to advertise a certain reformulation of general relativity, closely related to Plebański formulation, recently studied in a series 
of papers by the present author. In this formulation GR is described not as BF theory with constraints, but rather as BF theory with a potential for the B-field. It is arguably simpler to deal with potentials than with constraints, both in the continuum or discrete settings. Thus, the reformulation of GR described in this paper may shed new light on the problem of quantization of self-dual Plebański gravity. We also argue that it may be of help to understand the question of renormalization in the spin foam context.

\section{Self-dual Plebański formulation}

Before we describe a generalization of Plebański theory that converts it into a BF theory plus potential let us briefly describe the original, self-dual formulation 1 of Plebański with constraints. There are some excellent expositions of this formulation, see e.g. $\frac{5}{5}$, where we send the reader for more details.

The main idea behind Plebański's formulation of GR is the observation that the Einstein condition $R_{\mu \nu} \sim g_{\mu \nu}$, where $R_{\mu \nu}$ is the Ricci tensor of the metric $g_{\mu \nu}$ can be stated as the condition that the self-dual part of the Riemann curvature tensor

$R_{\mu \nu \rho \sigma}$ with respect to first pair of indices is also self-dual with respect to the second pair. Thus, let us introduce the notion of Hodge operator as acting on two forms $X_{\mu \nu}$ and sending $X_{\mu \nu} \rightarrow X_{\mu \nu}^{*}=(1 / 2) \epsilon_{\mu \nu}^{\rho \sigma} X_{\rho \sigma}$, where $\epsilon_{\mu \nu \rho \sigma}$ is the volume form of the metric $g_{\mu \nu}$, and the indices are raised and lowered using the metric. Using the Hodge operator one can introduce the projectors $P^{ \pm}=(1 / 2)(\operatorname{Id} \pm(1 / i) *)$ onto self- and anti-self dual two-forms. The Einstein condition can then be checked to be equivalent to, schematically:

$$
P^{+} R P^{-}=0,
$$

where $R$ is the Riemann curvature tensor. Let now $A$ be the restriction of the LeviCivita connection for $g_{\mu \nu}$ to the bundle of self-dual two-forms. The condition can be stated as a requirement that the curvature of $A$ is self-dual as a two-form. This observation (known in the mathematical literature as the Atiyah-Hitchin-Singer theorem [6] is a way to rephrase Plebański's formulation [1]. Interestingly, Plebański's paper was published one year earlier than 6 .

More concretely, in Plebański's theory one starts with an $\mathrm{SO}(3)$ principal bundle $P$ over the spacetime $M$ (complexified $\mathrm{SO}(3)$ in the case one wants to describe Lorentzian signature spacetimes). Here $M$ is a manifold without any additional structure such as a metric on it. The metric will appear later, only indirectly constructed from other basic objects of the theory. In physical applications $M$ is noncompact, and so the bundle $P$ is trivial. Let then $A$ be a connection in the associated bundle. In addition to the associated bundle, whose fibers are copies of the Lie algebra $\mathfrak{s} u(2)$ of $\mathrm{SO}(3)$, one can construct more interesting bundles by tensoring it with the bundles of forms on $M$. Of particular interest for Plebański theory is the bundle of Lie-algebra valued two-forms. Let $B$ be a section of this bundle. This is the object that will later receive the interpretation of carrying information about the metric. 
The connection $A$ defines a derivative operator $D_{A}$ that can be naturally extended to act on Lie-algebra-valued forms, in particular on $B$. One of the basic equations of Plebański theory then states:

$$
D_{A} B=0,
$$

which can be rephrased by saying that the two-form field $B$ is "covariantly constant" with respect to $A$. As it can be shown, given $B$ this uniquely determines $A$ (provided $B$ satisfies some non-degeneracy condition).

The second main equation of Plebański theory states that the curvature $F=$ $d A+(1 / 2) A \wedge A$ of $A$, which is a two-form with values in the Lie algebra of $\mathrm{SO}(3)$, can be decomposed purely into the two-forms $B$. Thus, the equation states that there exists some endomorphism $\Phi$ of the Lie algebra $\mathfrak{s} u(2)$ such that:

$$
F=\Phi(B), \quad \Phi \in \operatorname{End}_{\mathfrak{s} u(2)} .
$$

To see that this equation is non-vacuous note that a general two-form, when expanded into a basis of two-forms, has six components. However, the $\mathfrak{s} u(2)$-valued two-form $B$ spans only a three-dimensional space in the space of two-forms, and so not any two-form is of the form (3).

The third equation of Plebański theory is a condition on the two-form field $B$, which, in a sense, relates this object to a spacetime metric. Indeed, being an $\mathfrak{s} u(2)$-valued two-form, the field $B$ needs $6 \times 3$ numbers to be described. However, a spacetime metric has only 10 components. At the same time, 3 of the 18 components of $B$ are "gauge" and can be eliminated by the action of $\mathrm{SO}(3)$. This means that if $B$ is to describe a spacetime metric it must satisfy some 5 additional equations. These are as follows. Consider a four-form $B \wedge_{\otimes} B$. This is a four-form with values in the symmetric tensor product $(\mathfrak{s} u(2) \otimes \mathfrak{s} u(2))_{s}$. However, on $(\mathfrak{s} u(2) \otimes \mathfrak{s} u(2))_{s}$ we have an $\mathrm{SO}(3)$-invariant object - the Killing-Cartan form Id, or, in other words, the $\mathrm{SO}(3)$-invariant metric on $\mathfrak{s} u(2)$. A natural equation on $B$ arises if one requires $B \wedge B$ to be proportional to Id:

$$
B \wedge_{\otimes} B \sim \mathrm{Id},
$$

where the proportionality coefficient is an arbitrary four-form and can be determined by taking the trace of this equation. The equation (4) is known as the simplicity or metricity condition on $B$, and, as is not hard to see, is actually a set of 5 equations. Thus, it removes 5 out of the 18 components of $B$, leaving 10 plus the gauge 3 , which is the correct number to describe a spacetime metric.

The spacetime metric itself arises by observing that a non-degenerate (i.e. linearly independent) triple of two-forms can be declared to span the space of self-dual two-forms, and this defines the notion of self-duality on two-forms. This is, in turn, equivalent to a conformal metric, as is shown in e.g. 7 . What was shown by Plebański 1 is that when $B$ satisfies (4) then the arising metric is unique. Moreover, in this case the connection $A$ satisfying (2) turns out to be just the self-dual part of the 
Levi-Civita metric-compatible connection, and then (3) is just the equation (1) in disguise.

All the above equations can be obtained from a rather simple and natural action principle. Thus, let us introduce:

$$
S[B, A, \Phi]=\int_{M} \operatorname{Tr}\left(B \wedge F-\frac{1}{2} B \wedge \Phi(B)\right) .
$$

Here $\mathrm{Tr}$ is the Killing-Cartan symmetric bilinear pairing on $\mathfrak{s u}(2)$ that above, as an element of $\left(\mathfrak{s} u^{*}(2) \otimes \mathfrak{s} u^{*}(2)\right)_{s} \sim(\mathfrak{s} u(2) \otimes \mathfrak{s} u(2))_{s}$ we have denoted by Id. Varying (5) with respect to $A$ one gets (2), varying it with respect to $B$ one gets (3), and finally varying the action with respect to $\Phi$, which in (5) can be seen to be necessarily symmetric, one obtains (4). Note that to get (4) only the traceless part of the matrix $\Phi$ must be varied. The trace part is (proportional to) the cosmological constant. This finishes our short description of the Plebański theory.

In its form (5) the theory is that of BF type, i.e. its action is given by that of $B F$ theory - the first term in (5), plus an extra non-derivative term for the $B$ field. Such theories are thought to be susceptible to the spin foam quantization methods. However, it was so far not possible to understand how the constraints (4) must be taken care of in the spin foam formalism. For this reason no sufficiently developed spin foam model motivated by (5) exists, see, however, $\frac{4}{\text {. }}$

\section{Deformations of general relativity}

As may have become clear from the discussion of the previous section, the simplicity condition (44) is only used to restrict the possible two-form fields $B$ considered in the other equations (2), (3). However, it was also pointed out that an arbitrary two-form field $B$ can be used to define a conformal metric. Thus, ignoring the issue with the conformal factor for the moment, one could also consider a gravity theory given by the equations (2), (3) without (4) imposed. It turns out that the Bianchi identities derivable from (2), (31) constrain the system sufficiently to obtain a closed system of equations, see 8 for a demonstration of this. Thus, dropping the simplicity constraints (4) one arrives at the following theory:

$$
S[B, A]=\int_{M} \operatorname{Tr}(B \wedge F)-\frac{1}{2} V(B \wedge B) .
$$

Here $V(\cdot)$ is an arbitrary gauge-invariant potential function, which, in order for the action to make sense, is required to be a homogeneous function of order one in its $(\mathfrak{s} u(2) \otimes \mathfrak{s} u(2))_{s}$ tensor four-form valued argument. Indeed, the matrix-valued four-form $B \wedge B$ can always be written as some four-form times a matrix, and then the four-form can be pulled out of the potential function using its homogeneity. The result can then be integrated over $M$ to produce a scalar. The homogeneity of $V$ then guarantees that this does not depend on which four-form is used.

The field equations following from (6) are essentially unchanged from (2), (3). Thus, when varying the action with respect to $A$ one gets (2). When varying the 
action with respect to the two-form field one gets (3), with the only novelty being that the endomorphism $\Phi$ that in Plebański theory was left unspecified, is now determined by the two-form field itself, via the potential $V$. Thus, we get:

$$
F=\frac{1}{2} \frac{\partial V}{\partial B}
$$

where this equation makes sense in view of the homogeneity of the potential.

The action (6) defines a much larger class of theories than (5), for the potential $V(\cdot)$ may be quite arbitrary. In particular, the new class of theories is parametrized by an infinite number of the "coupling constants" - e.g. the coefficients of the decomposition of $V(\cdot)$ into a power series. It is not hard to show, see e.g. 8 for details, that when the potential $V(\cdot)$ becomes infinitely steep (in an appropriate sense) then one effectively imposes (44) and recovers GR. For a generic potential the theory (6) is, however, distinct from general relativity. In spite of this, it can be shown quite easily, see e.g. 9, that the theory in question still propagates just two graviton polarizations, exactly as in GR. Thus, the class of theories (6) provides an infinite parameter family of deformations of GR, with the Einstein's theory easily recoverable from (6) via a simple limit. The question of how to recover a preferred, physical metric from the conformal class of metrics naturally described by (6) has also been recently resolved in 10 .

The infinite-parameter class of theories (6) was first written down in 11, generalizing an earlier work by Capovilla (later published as 12 ) that studied a oneparameter family of deformations dubbed "neighbors of GR" by the author. Both 11 and 12 describe deformations using the so-called pure connection formulation of GR 13. Thus, it would be rather hard to recognize (6) in the results of 11 and 12 . The same theory was later rediscovered in 14 starting directly from the Plebański formulation. The equivalence between the two descriptions was demonstrated in 15 . The formulation described here (with a potential for the two-form field) is spelled out in more details in e.g. 8 .

\section{Quantum Gravity}

We would like to argue that it is the class (지 - not general relativity (5) - that should be used as a natural starting point for the spin foam (and possibly perturbative) quantization of gravity.

First, the theory in its version (6) does not contain any additional "Lagrange multiplier" fields such as $\Phi$ in (5). For this reason it is likely to be much easier to deal with in the discrete spin foam setting, where we already have a good deal of experience with working with the discrete versions of the $B$ and connection fields. Thus, it seems likely that the class of theories (6) is amenable to the spin foam quantization techniques rather directly. It just remains to be determined how to translate the property of the potential being a homogeneous function of degree one into the discrete setting. 
The second point we would like to make may initially sound a bit unconventional from the spin foam perspective. The point is that Einstein's theory, given in Plebański formulation by (5), is non-renormalizable. Thus, one does not expect this theory to play any direct role in the Planckian regime that we would like our quantum gravity to describe. If anything, one should expect that the relevant Planck scale theory should be a modified one, taking into account the quantum corrections that become essential at high energies. Such a much larger class of theories that takes into account at least some (if not all) the possible corrections is given by (6). Indeed, its original motivation in 14 was precisely to come to terms with the renormalization in quantum gravity.

Translated into the language of spin foams this renormalization motivation may be formulated as follows. In spin foam approach to quantum gravity one obtains an amplitude for a manifold by "gluing" together amplitudes for the individual spacetime simplices, see e.g. 3 and references therein for more details. Let us consider the "renormalization" in the context of spin foams, i.e. analyze what happens when one computes the simplex $\sigma$ amplitude as the result of integration over the labels of the "smaller" simplices that are glued together to make $\sigma$ (in a technical jargon this corresponds to an e.g. $5 \rightarrow 1$ move). When the elementary simplex amplitudes are built as dictated by the Plebański action (5) (or its $\mathrm{SO}(4)$ version), the new simplex amplitude - the result of the spin foam "renormalization group flow" - is of a different type, not anymore describable as coming from the original Plebański action. This is, we believe, how the non-renormalizability of GR manifests itself in the spin foam context. Thus, the spin foam renormalization group flow does not preserve the classical action (5) one starts from. As we have already said, we find this entirely natural, and having to do with the non-renormalizability of the underlying theory.

It is however possible (but quite non-trivial to show) that some larger class of theories may be closed under such a renormalization group flow. In the discrete setting of spin foams this would manifest itself in the simplex amplitude given by the result of the $5 \rightarrow 1$ move being of the same type as one started from, but with all the coupling constants - parameters of the theory - being changed in some subtle way. Should one find the class of theories with such a property, one can then see whether its UV completion exists by determining whether there is some non-trivial UV fixed point of the flow. This fixed point, if exists, would then provide the sought UV theory. It is then clear that the first step in the direction of this program is to enlarge the class of gravity theories that is being considered. We would like to propose the class (6) as a viable and natural arena for these ideas in the spin foam context.

\section{Acknowledgments}

The author was supported by an EPSRC Advanced Fellowship. 


\section{References}

1. J. F. Plebański, "On the separation of Einsteinian substructures," J. Math. Phys. 18, 2511 (1977).

2. R. De Pietri and L. Freidel, "so(4) Plebanski Action and Relativistic Spin Foam Model," Class. Quant. Grav. 16, 2187 (1999) arXiv:gr-qc/9804071.

3. L. Freidel and K. Krasnov, "A New Spin Foam Model for 4d Gravity," Class. Quant. Grav. 25, 125018 (2008) arXiv:0708.1595 [gr-qc]].

4. M. P. Reisenberger, "A left-handed simplicial action for euclidean general relativity," Class. Quant. Grav. 14, 1753 (1997) arXiv:gr-qc/9609002.

5. R. Capovilla, T. Jacobson, J. Dell and L. Mason, "Selfdual two forms and gravity," Class. Quant. Grav. 8 (1991) 41.

6. M. F. Atiyah, N. J. Hitchin and I. M. Singer, "Selfduality In Four-Dimensional Riemannian Geometry," Proc. Roy. Soc. Lond. A 362, 425 (1978).

7. T. Dray, R. Kulkarni and J. Samuel, "Duality and conformal structure," Journ. Math. Phys. 30, 1306 (1989).

8. K. Krasnov, "Plebanski gravity without the simplicity constraints," Class. Quant. Grav. 26, 055002 (2009) arXiv:0811.3147 [gr-qc]].

9. K. Krasnov, "On deformations of Ashtekar's constraint algebra," Phys. Rev. Lett. 100, 081102 (2008) arXiv:0711.0090 [gr-qc]].

10. K. Krasnov, "Motion of a 'small body' in non-metric gravity," arXiv:0812.3603 [gr-qc].

11. I. Bengtsson, "The Cosmological constants," Phys. Lett. B 254, 55 (1991).

12. R. Capovilla, "Generally Covariant Gauge Theories," Nucl. Phys. B 373, 233 (1992).

13. R. Capovilla, T. Jacobson and J. Dell, "A Pure spin connection formulation of gravity," Class. Quant. Grav. 8, 59 (1991).

14. K. Krasnov, "Renormalizable Non-Metric Quantum Gravity?," arXiv:hep-th/0611182

15. I. Bengtsson, "Note on non-metric gravity," Mod. Phys. Lett. A 22, 1643 (2007) arXiv:gr-qc/0703114. 